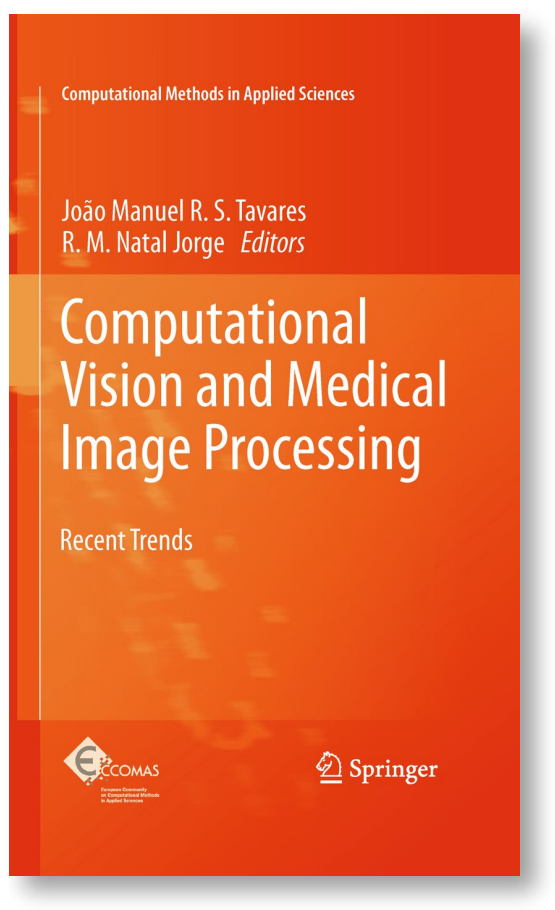

2011, IX, 349 p.

\section{Printed book}

\section{Hardcover}

- 139,99€|£126.00|\$189.00

- * $149,79 €(D)|153,99 €(A)|$ CHF 186.50

eBook

\section{Available from your library or}

- springer.com/shop

my My Copy

\section{Printed eBook for just}

- $\mid \$ 24.99$

- springer.com/mycopy
J. Tavares, R.M.N. Jorge (Eds.)

\section{Computational Vision and Medical Image Processing}

Recent Trends

Series: Computational Methods in Applied Sciences, Vol. 19

- Contains extended versions of papers presented to the International Conference VIPIMAGE 2009. This conference was the second ECCOMAS thematic conference on computational vision and medical image-processing

- It covers topics related to image processing and analysis, medical imaging and computational modeling and simulation, and considers their multidisciplinary nature

This book contains extended versions of papers presented at the international Conference VIPIMAGE 2009 - ECCOMAS Thematic Conference on Computational Vision and Medical Image, that was held at Faculdade de Engenharia da Universidade do Porto, Portugal, from 14th to 16th of October 2009. This conference was the second ECCOMAS thematic conference on computational vision and medical image processing. It covered topics related to image processing and analysis, medical imaging and computational modelling and simulation, considering their multidisciplinary nature.

The book collects the state-of-the-art research, methods and new trends on the subject of computational vision and medical image processing contributing to the development of these knowledge areas. 


\section{Preface}

Nowadays, computational methodologies of signal processing and imaging analysis for 2D, 3D and even 4D data are commonly used for various applications in society. For example, Computational Vision systems are progressively used for surveillance tasks, traffic analysis, recognition process, inspection purposes, human-machine interfaces, 3D vision and deformation analysis.

One of the main characteristics of the Computational Vision domain is its intermultidisciplinary nature. In fact, in this domain, methodologies of several other fundamental sciences, such as Informatics, Mathematics, Statistics, Psychology, Mechanics and Physics are regularly used. Besides this inter-multidisciplinary characteristic, one of the main rationale that promotes the continuous effort being made in this area of human knowledge is the number of applications in the medical area. For instance, statistical or physical procedures on medical images can be used in order to model the represented structures. This modelling can have different goals, for example: shape reconstruction, segmentation, registration, behavioural interpretation and simulation, motion and deformation analysis, virtual reality, computer-assisted therapy or tissue characterization.

The main objective of the ECCOMAS Thematic Conferences on Computational Vision and Medical Image Processing (VIPimage) is to promote a comprehensive forum for discussion on the recent advances in the related fields and try to identify areas of potential collaboration between researchers of different sciences.

This book contains the extended versions of nineteen papers selected from works presented at the second ECCOMAS thematic conference on Computational Vision and Medical Image processing (VIPimage 2009), which was held at the Engineering Faculty of the University of Porto, Portugal. It gathers together the state-of-the-art on the subject of Computational Vision and Medical Image processing contributing to the development of these knowledge areas and showing new trends in these fields.

The Editors would like to take this opportunity to thank to the European Community on Computational Methods in Applied Sciences, the Portuguese Association of Theoretical, Applied and Computational Mechanics, the University of Porto, all sponsors, all members of the International Scientific Committee and to all Invited Lecturers and Authors.

Faculty of Engineering

University of Porto, Portugal
João Manuel R.S. Tavares

R.M. Natal Jorge 


\section{Contents}

Automatic Segmentation of the Optic Radiation Using DTI

in Healthy Subjects and Patients with Glaucoma .....................

Ahmed El-Rafei, Tobias Engelhorn, Simone Waerntges,

Arnd Doerfler, Joachim Hornegger, and Georg Michelson

Real Time Colour Based Player Tracking in Indoor Sports

Catarina B. Santiago, Armando Sousa, Luís Paulo Reis, and Maria Luísa Estriga

Visualization of the Dynamics of the Female Pelvic Floor

Reflex and Steady State Function

Christos E. Constantinou, Qiyu Peng, and Sadao Omata

Population Exposure and Impact Assessment: Benefits of Modeling Urban Land Use in Very High Spatial and Thematic Detail

Christoph Aubrecht, Mario Köstl, and Klaus Steinnocher

Dynamic Radiography Imaging as a Tool in the Design

and Validation of a Novel Intelligent Amputee Socket

George Papaioannou, Dimitris Tsiokos, Goeran Fiedler,

Christos Mitrogiannis, Ilya Avdeev, Jake Wood, and Ray McKinney

A Discrete Level Set Approach for Texture Analysis of Microscopic Liver Images

Daniela Iacoviello

Deformable and Functional Models

Demetri Terzopoulos 
Medical-GiD: From Medical Images to Simulations, 4D MRI

Flow Analysis

Eduardo Soudah, Julien Pennecot, Jorge S. Pérez, Maurizio

Bordone, and Eugenio Oñate

KM and KHM Clustering Techniques for Colour Image

Quantisation

Mariusz Frackiewicz and Henryk Palus

Caries Detection in Panoramic Dental X-ray Images

João Oliveira and Hugo Proença

Noisy Medical Image Edge Detection Algorithm

Based on a Morphological Gradient Using Uninorms

Manuel González-Hidalgo, Arnau Mir Torres, Daniel Ruiz

Aguilera, and Joan Torrens Sastre

Leveraging Graphics Hardware for an Automatic

Classification of Bone Tissue

Manuel Jesús Martín-Requena and Manuel Ujaldón

A Novel Template-Based Approach to the Segmentation

of the Hippocampal Region

M. Aiello, P. Calvini, A. Chincarini, M. Esposito, G. Gemme,

F. Isgrò, R. Prevete, M. Santoro, and S. Squarcia

\section{Model-Based Segmentation and Fusion of 3D Computed}

Tomography and 3D Ultrasound of the Eye

for Radiotherapy Planning.

M. Bach Cuadra, S. Gorthi, F.I. Karahanoglu, B. Paquier, A. Pica,

H.P. Do, A. Balmer, F. Munier, and J.-Ph. Thiran

Flow of a Blood Analogue Solution Through Microfabricated

Hyperbolic Contractions .265

P.C. Sousa, I.S. Pinho, F.T. Pinho, M.S.N. Oliveira, and M.A. Alves

Molecular Imaging of Hypoxia Using Genetic Biosensors

Pablo Iglesias and J.A. Costoya

Microscale Flow Dynamics of Red Blood Cells in Microchannels:

An Experimental and Numerical Analysis

R. Lima, C.S. Fernandes, R. Dias, T. Ishikawa, Y. Imai,

and T. Yamaguchi 
Two Approaches for Automatic Nuclei Cell Counting in Low

Resolution Fluorescence Images ....

Thierry Brouard and Aurélie Chantôme

Cerebral Aneurysms: A Patient-Specific and Image-Based

Management Pipeline

M.C. Villa-Uriol, I. Larrabide, J.M. Pozo, M. Kim, M. De Craene,

O. Camara, C. Zhang, A.J. Geers, H. Bogunović, H. Morales, and A.F. Frangi 


\title{
Microscale Flow Dynamics of Red Blood Cells in Microchannels: An Experimental and Numerical Analysis
}

\author{
R. Lima, C.S. Fernandes, R. Dias, T. Ishikawa, Y. Imai, and T. Yamaguchi
}

\begin{abstract}
The blood flow dynamics in microcirculation depends strongly on the microvascular networks composed with short irregular vessel segments which are linked by numerous bifurcations. This paper presents the application of a confocal micro-PTV system to track RBCs through a rectangular polydimethysiloxane (PDMS) microchannel with a bifurcation. By using a confocal micro-PTV system, we have measured the effect of bifurcation on the flow behaviour of both fluorescent particles diluted in pure water and RBCs in concentrated suspensions. After performing simulations with the commercial finite element software package POLYFLOW ${ }^{\circledR}$, some experimental results were compared with the numerical results and the limitations of these simulations were outlined.
\end{abstract}

Keywords Blood flow $\cdot$ Microvascular networks $\cdot$ Bifurcation · Microchannel

\footnotetext{
R. Lima (舟) and R. Dias

ESTiG, IPB, C. Sta. Apolonia, 5301-857 Braganca, Portugal

e-mail: ruimec@ipb.pt; ricardod@ipb.pt

and

CEFT, FEUP, R. Dr. Roberto Frias, 4200-465 Porto, Portugal

C.S. Fernandes

ESTiG, IPB, C. Sta. Apolonia, 5301-857 Braganca, Portugal

e-mail: cveiga@ipb.pt

T. Ishikawa and Y. Imai

Department of Bioengineering \& Robotics, Graduate School of Engineering,

Tohoku University, 6-6-01 Aoba, 980-8579 Sendai, Japan

e-mail: ishikawa@pfsl.mech.tohoku.ac.jp; yimai@pfsl.mech.tohoku.ac.jp

T. Yamaguchi

Department of Biomedical Engineering, Graduate School of Engineering,

Tohoku University, 6-6-01 Aoba, 980-8579 Sendai, Japan

e-mail: takami@pfsl.mech.tohoku.ac.jp
} 


\section{Introduction}

The phenomena of blood flow in microvessels (diameters less than $300 \mu \mathrm{m}$ ) are crucial in maintaining healthy organs and tissues. Although these phenomena have been studied for many years it still remains incompletely understood [25]. It is therefore important to investigate the behaviour of blood flow occurring at microvessels in order to better understand the role of blood cells in the process of delivering oxygen and materials to the organs and tissues.

The complexity to control and obtain reliable measurements of the blood flow behaviour through the in vivo microvascular system has led several researchers to perform their studies by using narrow glass tubes with simple geometries. By using in vitro models it allows a more precise control over the experimental variables of interest and extracts detailed information of the flow behaviour of individual blood cells. In fact, much of the understanding of the haemodynamics phenomena observed in microcirculation was obtained from studies on the both macro and microrheology properties of blood flowing through glass microtubes $[3,11,13,16,17,25]$.

Although glass microchannels present certain similarities to in vivo microcirculation, it is also clear that these kind of in vitro experiments differ from microvessels in several respects, such as: elasticity of microvessels, effect of the endothelial surface layer and microvascular networks composed with short irregular vessel segments which are linked by numerous bifurcations. Thus it was not surprising that several studies on blood flow in glass microtubes and in microvessels have yielded conflicting results with respect to blood viscosity [20] and flow resistance [27]. Hence, the rheological properties of blood should not be only interpreted from measurements in viscometers and microtubes with simple geometries.

In vivo microvascular networks are composed of many divergent microvascular bifurcations which likely influence the blood flow behaviour in the microcirculation. In the present work, a confocal micro-PTV system is used to measure the blood flow through a symmetric PDMS bifurcation fabricated by soft lithography. Moreover, the experimental data were compared numerically by using the commercial finite element software package POLYFLOW ${ }^{\circledR}$. By using this combination we expect to gain understanding about several important parameters that affect the blood flow through a diverging microvessel bifurcation.

\section{Materials and Methods}

\subsection{Fabrication of the Microchannels}

The microchannels will be manufactured in polydimethylsiloxane (PDMS) due to their advantageous properties, including good optical transparency and biocompatibility, easily reversible sealing to glass, elasticity, replication of fine and complex 


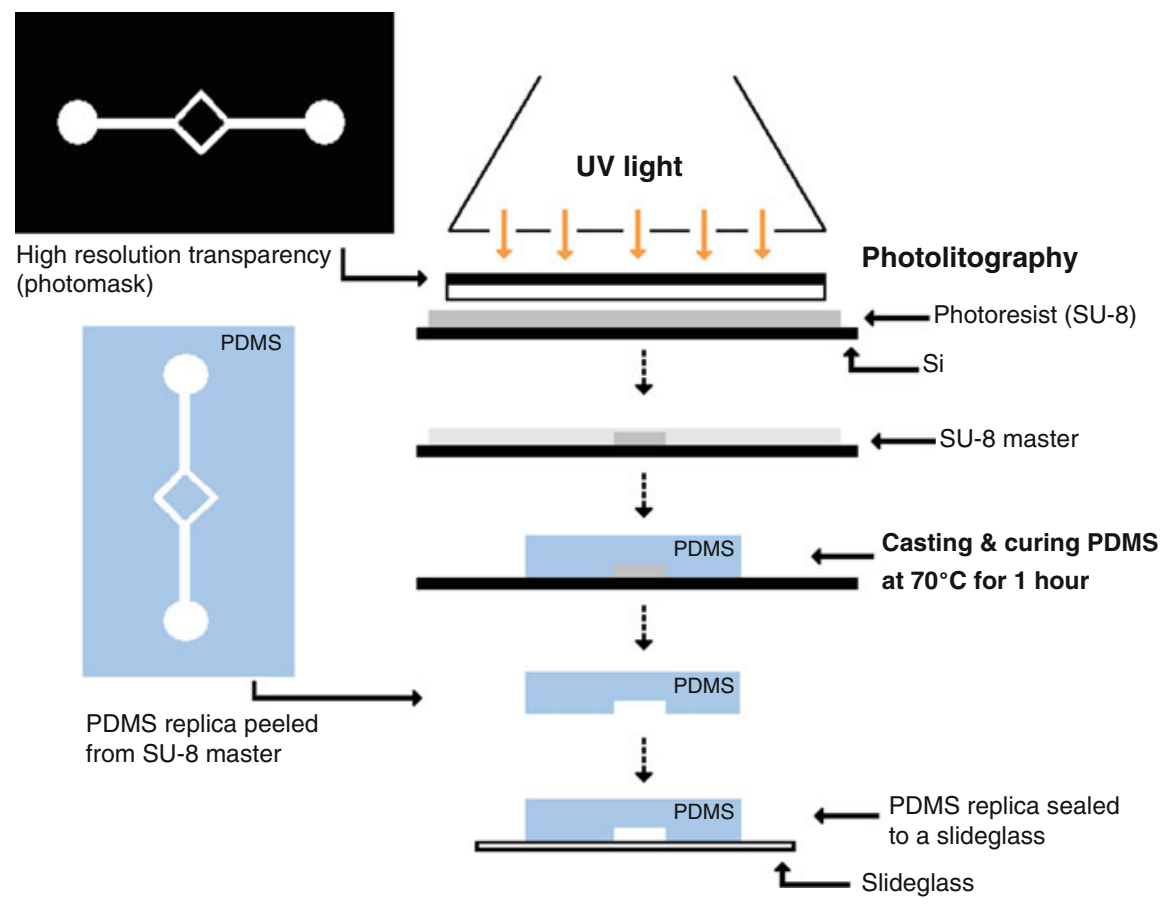

Fig. 1 Main steps of the soft lithographic technique to manufacture a microchannel with a symmetrical bifurcation geometry

geometries, and high gas permeability which is suitable for culturing cells inside the microchannels [17]. The procedure to manufacture the PDMS microchannel with a symmetric bifurcation is illustrated in Fig. 1. Briefly, the microchannel geometry was designed using a CAD system, and a high resolution $(25,400 \mathrm{dpi})$ photomask was printed on plastic (polyester) transparencies at CAD/Art Services, Inc. (Bandon, USA). By applying a photolithographic technique (see Fig. 1), a solid master was fabricated on a silicon ( $\mathrm{Si}$ ) wafer with an ultrathick photoresist (SU-8). In this process, by using a spin-coating technique, a thin layer of SU-8 photoresist was coated on $\mathrm{Si}$ wafer and then patterned by UV exposure through the photomask. Using a SU-8 developer, it was possible to obtain the desired mold master. Then, metal wire posts were placed on the SU-8 mold master to create the connection channels to the input/output ports. The PDMS was made by mixing a base and curing agent (Silpot 184; Dow Corning, USA) at a weight ratio of 10:1. After removing the bubbles created during mixing, the PDMS was poured over the mold master and baked on a hot plate for about $1 \mathrm{~h}$ at $70^{\circ} \mathrm{C}$. Both master and PDMS were then cooled to room temperature and the PDMS design and embedded metal wire posts were removed from the SU-8 master and PDMS, respectively. Finally, the three-dimensional PDMS microchannel structure was cleaned with ethanol and brought into contact with a clean slide glass, where a reversible seal 
was formed spontaneously. The input/output ports were made by means of a $200 \mu 1$ micro-pipette tip. This tip was inserted tightly into the connection channels where it exerts pressure on the PDMS and provides a liquid proof seal. Top tubes were also fitted tightly into the micro-pipette tip in order to deliver the working fluids from the syringe pump. Due to the elasticity of the connection tubes and conical shape of the tip, we did not observe any fluid leakage during our experiments [17].

\subsection{Working Fluids and Geometry of the Bifurcation}

Two working fluids were used in this study: pure water and dextran 40 (Dx40) containing about $14 \%$ (14Hct) of human red blood cells (RBCs). The blood was collected from a healthy adult volunteer, where ethylenediaminetetraacetic acid (EDTA) was added to prevent coagulation. The RBCs were separated from the bulk blood by centrifugation and aspiration and then washed twice with physiological saline (PS). The washed RBCs were labeled with a fluorescent cell tracker (CMDil, C-7000, Molecular Probes) and then diluted with Dx40 to make up the required RBCs concentration by volume. All blood samples were stored hermetical at $4{ }^{\circ} \mathrm{C}$ until the experiment was performed at controlled temperature of about $37^{\circ} \mathrm{C}$ [13].

The geometry and dimensions of the microchannel bifurcation is illustrated in Fig. 2. In the present study we decided to use the following dimensions: $150 \mu \mathrm{m}$ wide for parent vessel $\left(\mathrm{W}_{0}\right) ; 75 \mu \mathrm{m}$ wide for daughter vessel $\left(\mathrm{W}_{1}=\mathrm{W}_{2}=1 / 2 \mathrm{~W}_{0}\right)$ and $50 \mu \mathrm{m}$ deep.

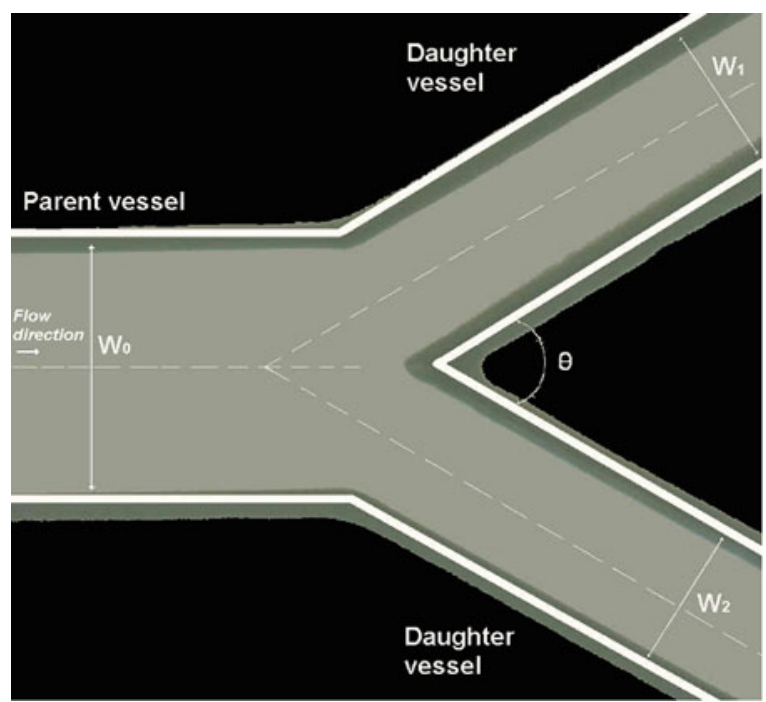

Fig. 2 Symmetrical bifurcation geometry used in this study: $\mathrm{W}_{0}=150 \mu \mathrm{m}, \mathrm{W}_{1}=\mathrm{W}_{2}=$ $75 \mu \mathrm{m}, \theta=60^{\circ}$, depth $=50 \mu \mathrm{m}$ 
Table 1 Experimental parameters used to calculate the $\operatorname{Re}$

\begin{tabular}{ll}
\hline Density $\left(\mathrm{kg} / \mathrm{m}^{3}\right)$ & 1,046 \\
Mean velocity $(\mathrm{m} / \mathrm{s})$ & $3.8 \times 10^{-4}$ \\
Hydraulic diameter $(\mathrm{m})$ & $7.5 \times 10^{-5}$ \\
Viscosity of Dx-40 $\left(\mathrm{Ns} / \mathrm{m}^{2}\right)$ & $4.5 \times 10^{-3}$ \\
$\mathrm{Re}$ & 0.007 \\
\hline
\end{tabular}

\subsection{Confocal Micro-PTV Experimental Set-Up}

The confocal micro-PTV system used in our experiment consists of an inverted microscope (IX71, Olympus, Japan) combined with a confocal scanning unit (CSU22, Yokogawa) and a diode-pumped solid state (DPSS) laser (Laser Quantum Ltd) with an excitation wavelength of $532 \mathrm{~nm}$. Moreover, a high-speed camera (Phantom v7.1) was connected into the outlet port of the CSU22. The microchannel was placed on the stage of the inverted microscope where the flow rate of the working fluids was kept constant ( $\mathrm{Re}=0.007)$ by means of a syringe pump (KD Scientific Inc.). The Reynolds number $(\mathrm{Re})$ and associated experimental parameters are summarized in Table 1. A thermo plate controller (Tokai Hit) was set to $37^{\circ} \mathrm{C}$. All the confocal images were captured in the middle of the microchannels with a resolution of $640 \times 480$ pixels, at a rate of 100 frames/s with an exposure time of $9.4 \mathrm{~ms}$. The recorded images were transferred to the computer and then evaluated in the Image $\mathbf{J}(\mathrm{NIH})$ [1] by using the manual tracking MtrackJ [21] plugin. As a result it was possible to track single RBCs through the middle plane of the microchannel (Fig. 3).

\subsection{Simulation Method}

The numerical calculations for the laminar isothermal flow of pure water were performed using the finite-element computational fluid dynamics (CFD) program POLYFLOW ${ }^{\circledR}$. The simulations were carried out in a $3 \mathrm{D}$ geometry representing the microchannel (see Fig. 4). The mesh used in the simulations was mainly constituted by quadrilateral elements, the discretization of the walls of the channel being presented in Fig. 4. The size of the elements was fixed after a grid independence test. The grids were successively refined and the velocity obtained with the different meshes were compared, the results being considered independent of the mesh when a difference bellow $1 \%$ was achieved [4, 8-10].

The equations solved were the conservation of mass and momentum equations for laminar incompressible flow of water. The problem is a non-linear problem, so it was necessary to use an iterative method to solve the referred equations. In order to evaluate the convergence of this process; a test based on the relative error in the velocity field was performed. For the velocity field, the modification on each node between two consecutive iterations is compared to the value of the velocity at the current iteration. In the present work, the convergence value was set to $10^{-4}$, since this value is appropriate for the studied problem [5, 8-10]. 


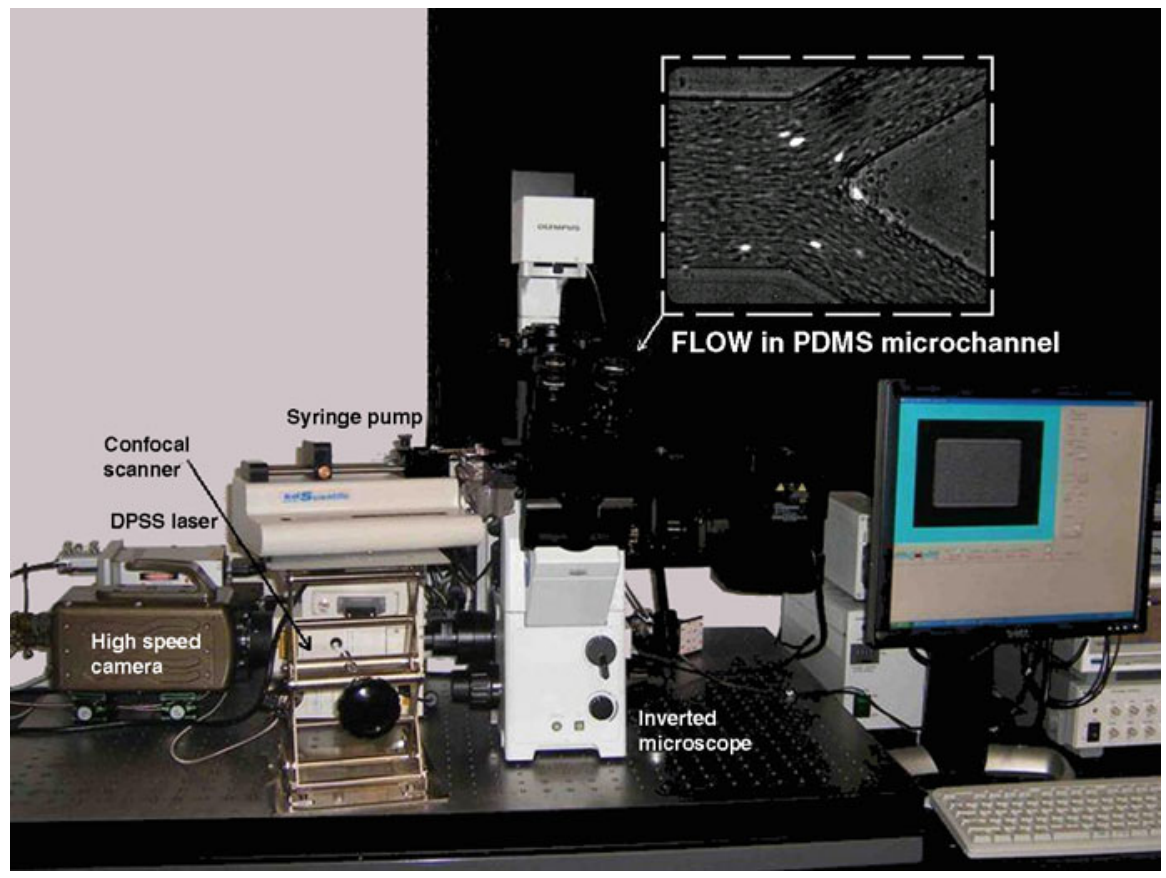

Fig. 3 Experimental set-up

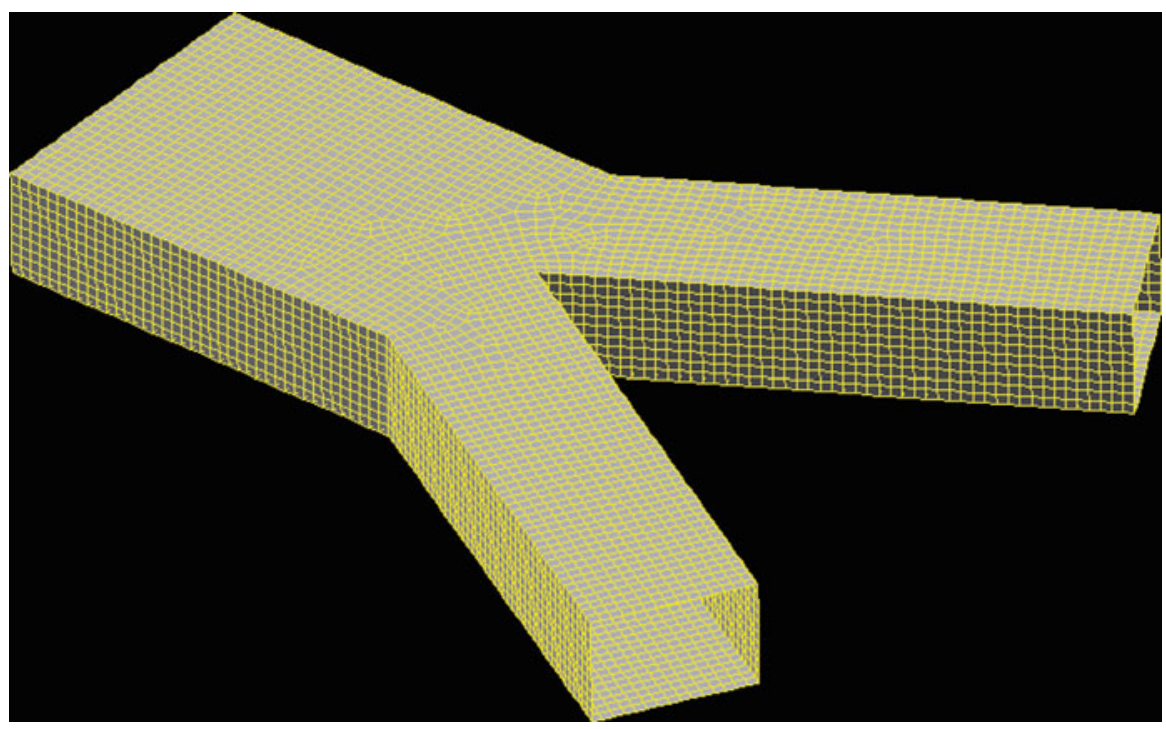

Fig. 4 Computational domain and mesh used 
Table 2 Rheological parameters of blood [12]

\begin{tabular}{lllllll}
\hline Rheological model & $\eta($ Pa.s $)$ & $K(-)$ & $n(-)$ & $\lambda(\mathrm{s})$ & $\eta_{\infty}(\mathrm{Pa} . \mathrm{s})$ & $\eta_{0}(\mathrm{~Pa} . \mathrm{s})$ \\
\hline Newtonian & 0.00345 & - & - & - & - & - \\
Power law model & - & 0.035 & 0.6 & - & - & - \\
Carreau model & - & - & 0.3568 & 3.313 & 0.00345 & 0.056 \\
\hline
\end{tabular}

The boundary conditions were established in order to reproduce the experimental conditions. The geometry of the bifurcation was idealized and close to the original dimensions of the photomask. Additionally, the mean velocity at the inlet of the microchannel was $3.8 \times 10^{-4} \mathrm{~m} / \mathrm{s}$ and slip at the walls of the channel was assumed to be non-existent. The referred velocity was imposed considering a constant flow rate on the referred boundary.

In the numerical study, blood was considered a Newtonian and non-Newtonian fluid In the last case, the rheology of the blood was described by two different constitutive models - power law model and the Carreau model [12] - which are, respectively, traduced mathematically by the equations:

$$
\begin{gathered}
\eta=K \dot{\gamma}^{n-1} \\
\eta=\eta_{\infty}+\left(\eta_{0}-\eta_{\infty}\right)\left[1+(\lambda \dot{\gamma})^{2}\right]^{(n-1) / 2},
\end{gathered}
$$

where $\eta$ is the viscosity of the fluid, $K$ the consistency index, $n$ the flow index behavior, $\dot{\gamma}$ the shear rate, $\eta_{\infty}$ the viscosity for high shear rates, $\eta_{0}$ the viscosity for low shear rates and $\lambda$ the natural time. For the blood, the rheological parameters present in the above equations are reported in Table 2.

\section{Results and Discussion}

The confocal micro-PIV system was first evaluated by comparing the experimental results not only with a well established analytical solution for steady flow in a rectangular microchannel [15] but also with a reliable numerical method that was used in past investigations to study the flow behaviour of Newtonian or non-Newtonian fluids at low Reynolds numbers [9, 10].

The numerical, experimental and analytical results of the present work were obtained for the middle plane ( $25 \mu \mathrm{m}$ height) of the rectangular microchannel. The averaged velocity data obtained from the confocal micro-PTV measurements, analytical solution and numerical simulation were in good agreement. A more detail description of these results can be found elsewhere [22].

By using a confocal micro-PTV system it was possible to obtain series of successive images at the middle of the bifurcation. Figures $5 \mathrm{a}$ and 6 show images with both fluorescent particles and labeled RBCs (laser-emitted light) flowing through a symmetric bifurcation, together with the correspondent time position tracking of both 

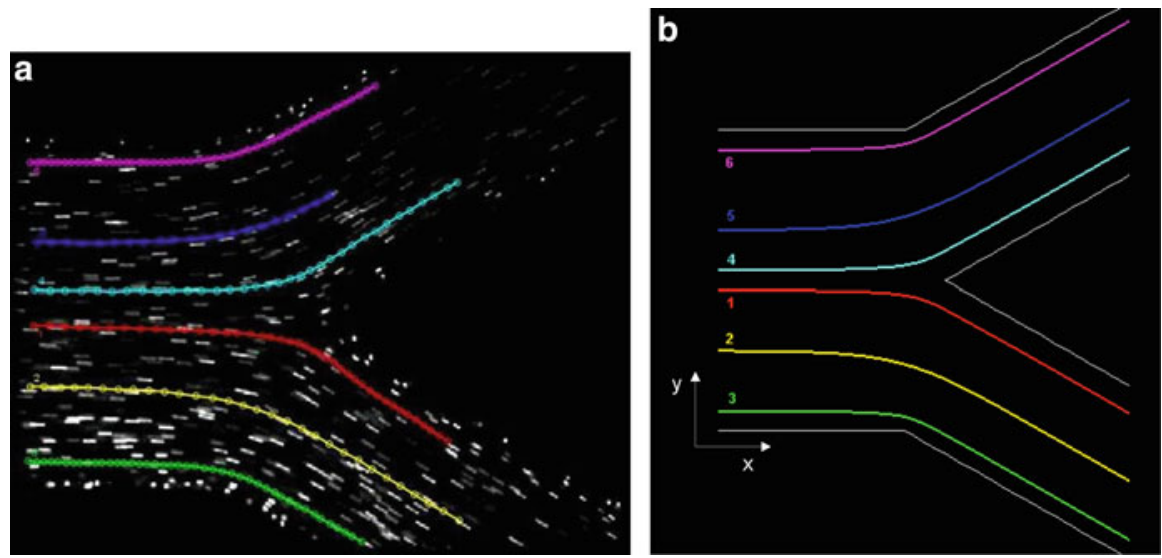

Fig. 5 (a) Paths displacement of fluorescent particles flowing in pure water; (b) Numerical trajectories using pure water

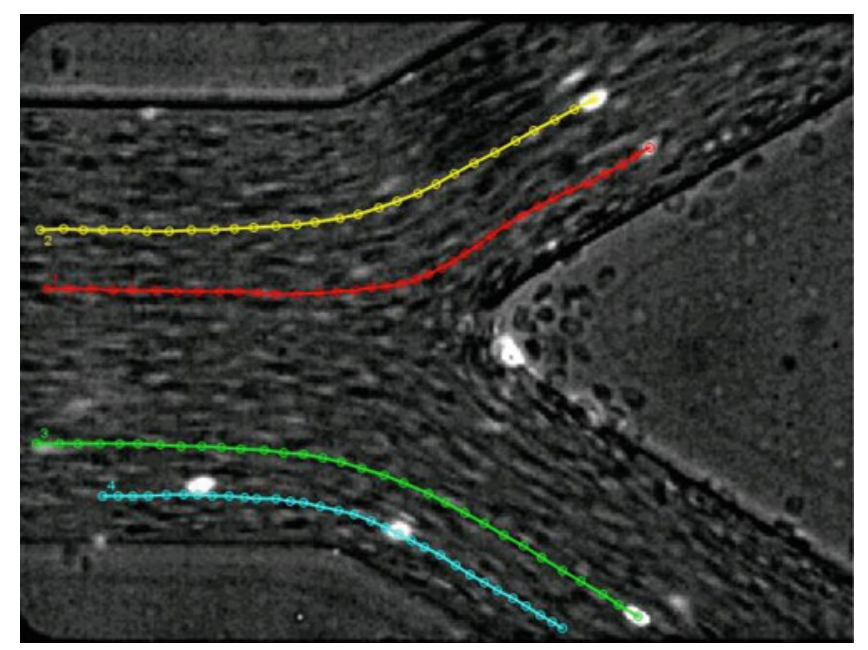

Fig. 6 Paths displacement of labeled RBCs (bright spots) flowing in physiological fluid with 14\% Hct $(32 \times)$

particles and individual RBCs. Qualitative comparison between the experimental data from pure water (see Fig. 5a) and the numerical simulation (see Fig. 5b), shows that in both cases the trajectories do not exhibit any appreciable deviations in the transversal ( $y y$ axis) direction.

In addition by comparing qualitatively the experimental data from both pure water and in vitro blood $(14 \% \mathrm{Hct})$ it is possible to observe that some RBC paths seems 


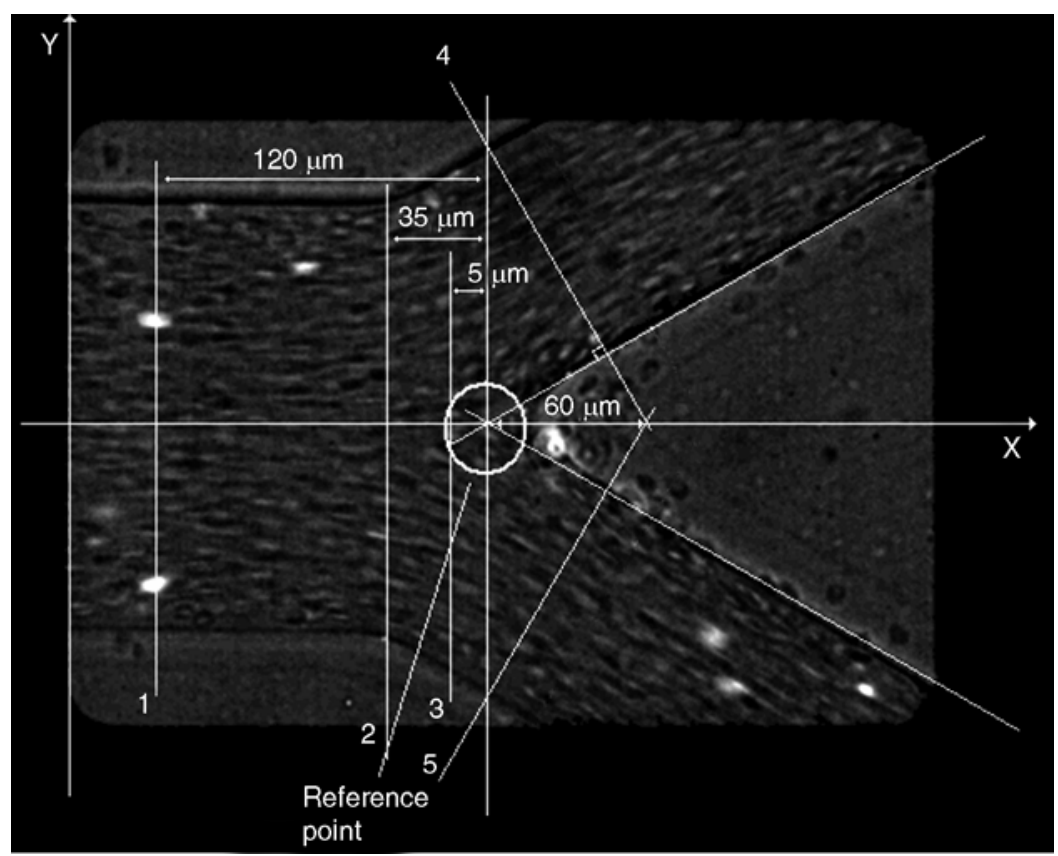

Fig. 7 Regions where the velocity profiles of the numerical and experimental results were compared

to suffer small deviations from the streamlines of the plasma flow probably due to flow perturbations caused by cell interactions in the neighbourhood of the apex of bifurcation.

Moreover numerical simulations of non-Newtonian models were performed around the bifurcation at the regions 1-5 (see Fig. 7).

Figures 8 and 9 show the velocity profiles for both computational and experimental results before and after the bifurcation, respectively.

Figures 8 and 9 suggest that the RBCs velocities close to the microchannel wall are higher than that obtained with the numerical models since slip at the walls of the channel was assumed to be non-existent in the latter case. It is well know, in microcirculation and other areas [4, 5, 13, 20, 28, 32], that RCBs, macromolecules, colloids, etc., are excluded from the region of the channel with low velocity and this may explain the observed differences.

Moreover, Figs. 8 and 9 confirm [35] that the velocity profiles obtained with the power law model $(n=0.6)$ are more flat than the ones obtained with the Newtonian model $(n=1)$. For infinite pseudoplasticity $(n=0)$ a plug flow should be obtained [35]. It is important to note that in Figs. 8 and 9 the average velocity obtained with the different constitutive equations was the same. 

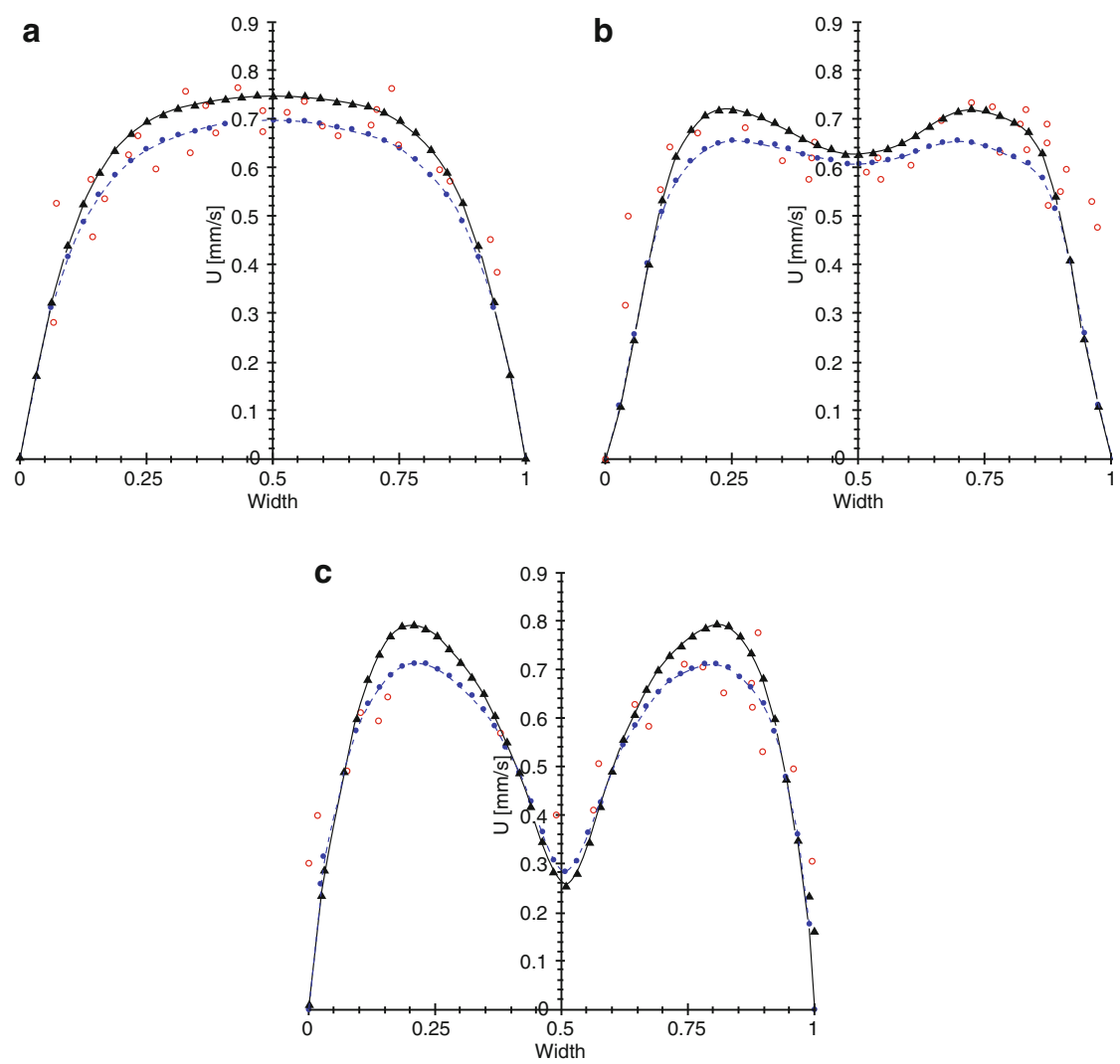

Fig. 8 Velocity profiles for both computational and experimental results before the bifurcation:

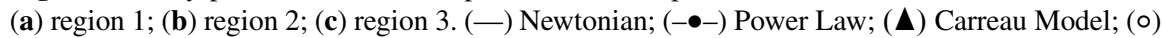
Confocal micro-PTV RBC velocities
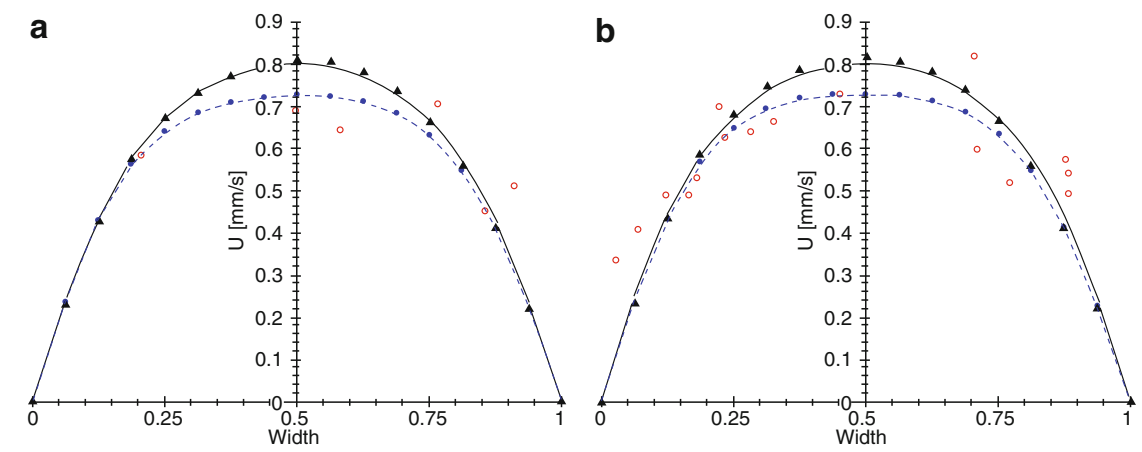

Fig. 9 Velocity profiles for both computational and experimental results after the bifurcation: (a)

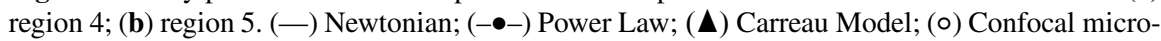
PTV RBC velocities 


\section{Limitations and Future Directions}

The primary goal of the present work is to provide new insights on the rheological properties of blood in microvascular network models. To accomplish it experimental flow studies was performed with a confocal micro-PTV system complemented with the most recent advances in microfabricated technologies. However, the soft-litography quality is strongly dependent not only on the photolitography methodology but also on the photomask resolution and its fidelity to the original design. In the present work we used a plastic photomask and according to our preliminary results we found small discrepancies between the tested in vitro model and the original design. Although these discrepancies were not taken into account in the present study we are aware about the possible influence of them on the results shown. Hence, in the future we are planning to carry on the current research by taking into account such discrepancies.

In vitro experimentation has the potential to provide a more realistic information on the flow properties of blood when compared with numerical simulations. However, once validated the numerical models can be a valuable tool to obtain more detailed insights about the blood rhelogical properties in microvascular networks. Generally, there are two major approaches to model of the non-Newtonian nature of blood [33]. One is based on the conventional continuum approach, in which a blood constitutive equation is assumed such as the casson model and the power-law model [36]. A more complex and realistic approach is based on a multiphase approach, in which the blood is considered as a multiphase suspension of deformable particles and where levels of submodeling for the behaviour of blood components are introduced. Some examples for this type of approach are the boundary element method [23, 26, 34], the immersed boundary method [2, 7, 24, 31], the lattice Boltzmann method $[6,29]$ and the moving particle semi-implicit (MPS) method $[19,30])$. Recent reviews on these numerical methods can be found in Liu et al. [18], Yamaguchi et al. [33] and Lima et al. [14]. Although the multiphase flow approach is a very promising method it requires massive computational power. Hence, only recently this latter approach is being actively pursued due to the advances of the computational techniques and the computing power.

In the current study we have only performed simulations by using a continuum computational approach to model of the non-Newtonian nature of blood. However for the size of the microcahnnel used in presented work we can not neglect the effect of the suspension of deformable cells on its flow behaviour. Hence, we expect in a near future to compare the obtained experimental results with multi-phase numerical models.

\section{Conclusions}

A confocal micro-PTV system for the analysis of the complex phenomena happening at bifurcations was proposed. The system was complemented by a softlitography technique to fabricate the in vitro models and by a continuum computational model approach to study the non-Newtonian nature of the working fluids. 
Qualitative experimental observations suggested that RBC paths around the bifurcation apex seems to suffer small deviations from the streamlines of the plasma flow probably due to cell interactions enhanced by the high local Hct originated at this region.

The simulations performed with a finite-element computational fluid dynamics (CFD) program POLYFLOW emphasized the need of developing a multiphase approach.

Acknowledgements This study was supported in part by the following grants: Grant-in-Aid for Science and Technology (BII/UNI/0532/EME/2008, PTDC/SAU-BEB/108728/2008, PTDC/SAUBEB/105650/2008 and PTDC/EME-MFE/099109/2008) from the Science and Technology Foundation (FCT) and COMPETE, Portugal and Grant-in-Aid for Scientific Research (S) from the Japan Society for the Promotion of Science (JSPS; No.19100008). We also acknowledge the support from the 2007 Global COE Program "Global Nano-Biomedical Engineering Education and Research Network". The authors would like also to thank Dr. C. Balsa for his valuable assistance and support for the MATLAB numerical calculations and Ms. B. Oliveira, Ms. D. Cidre and Mr. M. Lagoela for their valuable technical assistance in this research work.

\section{References}

1. Abramoff, M., Magelhaes, P., Ram, S.: Image processing with image J. Biophotonics Int. 11, 36-42 (2004)

2. Bagchi, P.: Mesoscale simulation of blood flow in small vessels. Biophys. J. 92, 1858-1877 (2007)

3. Chien, S., Usami, S., Skalak, R.: Blood flow in small tubes In: Renkins, M., Michel, C.C. (eds.) Handbook of Physiology-The Cardiovascular System IV, pp. 217-249. American Physiological Society, Bethesda (1984)

4. Dias, R.P.: Size fractionation by slalom chromatography and hydrodynamic chromatography. Recent Patents Eng. 2, 95-103 (2008)

5. Dias, R.P., Fernandes, C.S., et al.: Starch analysis using hydrodynamic chromatography with a mixed-bed particle column, Carbohydr. Polym. 74, 852-857 (2008)

6. Dupin, M.M., Halliday, I., et al.: Modeling the flow of dense suspensions of deformable particles in three dimensions. Phys. Rev. E. 75, 066707 (2007)

7. Eggleton, C.D., Popel, A.S.: Large deformation of red blood cell ghosts in a simple shear flow. Phys. Fluids. 10, 1834-1845 (1998)

8. Fernandes, C.S., Dias, R.P., et al.: Simulation of stirred yoghurt processing in plate heat exchangers. J. Food Eng. 69, 281-290 (2005)

9. Fernandes, C.S., Dias, R.P., et al.: Laminar flow in chevron-type plate heat exchangers: CFD analysis of tortuosity, shape factor and friction factor. Chem. Eng. Process.: Process Intensif. 46, 825-833 (2007)

10. Fernandes, C.S., Dias, R.P., et al.: Friction factors of power-law fluids in plate heat exchangers. J. Food Eng. 89, 441-447 (2008)

11. Goldsmith, H., Turitto, V.: Rheological aspects of thrombosis and haemostasis: Basic principles and applications. ICTH-Report-Subcommittee on Rheology of the International Committee on Thrombosis and Haemostasis. Thromb. Haemost. 55, 415-435 (1986)

12. Johnston, B.M., Johnston, P.R., et al.: Non-Newtonian blood flow in human right coronary arteries: Steady state simulations. J. Biomech. 37, 709-720 (2004)

13. Lima, R., Ishikawa, T., et al.: Measurement of individual red blood cell motions under high hematocrit conditions using a confocal micro-PTV system. Ann. Biomed. Eng. 37, 1546-1559 (2009) 
14. Lima, R., Ishikawa, T., et al.: Blood flow behavior in microchannels: Advances and future trends. In: Single and Two-Phase Flows on Chemical and Biomedical Engineering. Bentham (in press) (2011)

15. Lima, R., Wada, S., et al.: Confocal micro-PIV measurements of three dimensional profiles of cell suspension flow in a square microchannel. Meas. Sci. Tech. 17, 797-808 (2006)

16. Lima, R., Wada, S., et al.: In vitro confocal micro-PIV measurements of blood flow in a square microchannel: The effect of the haematocrit on instantaneous velocity profiles. J. Biomech. 40, 2752-2757 (2007)

17. Lima, R., Wada, S., et al.: In vitro blood flow in a rectangular PDMS microchannel: Experimental observations using a confocal micro-PIV system. Biomed. Microdevices 2(10), 153-167 (2008)

18. Liu, W.K., Liu, Y., et al.: Immersed finite element method and its applications to biological systems. Comput. Methods Appl. Eng. 195, 1722-1749 (2006)

19. Kondo, H., Imai, Y., et al.: Hemodynamic analysis of microcirculation in malaria infection. Ann. Biomed. Eng. 37, 702-709 (2009)

20. Maeda, N.: Erythrocyte rheology in microcirculation. Jpn. J. Physiol. 46, 1-14 (1996)

21. Meijering, E., Smal, I., Danuser, G.: Tracking in molecular bioimaging. IEEE Signal Process. Mag. 3(23), 46-53 (2006)

22. Oliveira, B., Lagoela, M., et al.: Analyses of the blood flow in a microchannel with a bifurcation. In: Proceedings of $3^{\circ}$ Congresso Nacional de Biomecânica, Bragança, Portugal (2009)

23. Omori, T., Ishikawa, T. et al.: Behavior of a red blood cell in a simple shear flow simulated by a boundary element method, In: Proceedings of Bioengineering 08, London, UK (2008)

24. Peskin, C.S.: Numerical analysis of blood flow in the heart. J. Comput. Phys. 25, 220-233 (1977)

25. Popel, A., Johnson, P.: Microcirculation and hemorheology. Annu. Rev. Fluid Mech. 37, 43-69 (2005)

26. Pozrikidis, C.: Numerical simulation of the flow-induced deformation of red blood cells. Ann. Biomed. Eng. 31, 1194-1205 (2003)

27. Pries, A., Secomb, T., et al.: Resistance to blood flow in microvessels in vivo. Circ. Res. 75, 904-915 (1994)

28. Small, H.: Hydrodynamic chromatography a technique for size analysis of colloidal particles. J. Colloid. Interface Sci. 48, 147-161 (1974)

29. Succi, S.: The Lattice Boltzmann Equation for Fluid Mechanics and Beyond, Clarendon Press, Oxford (2001)

30. Tsubota, K., et al.: Particle method for computer simulation of red blood cell motion in blood flow. Comp. Methods Programs Biomed. 83, 139-146 (2006)

31. Univerdi, S.O., Tryggvason, G.: A front-tracking method for viscous, incompressible multifluid flows. J. Comput. Phys. 100, 25-37 (1992)

32. Venema, E., Kraak, J.C., et al.: Packed-column hydrodynamic chromatography using $1-\mu \mathrm{m}$ non-porous silica particles. J. Chromatogr. A 740, 159-167 (1996)

33. Yamaguchi, T., Ishikawa, T., et al.: Computational blood flow analysis - new trends and methods. J. Biomech. Sci. Eng. 1, 29-50 (2006)

34. Youngren, G.K., Acrivos, A.: Stokes flow past a particle of arbitrary shape: A numerical method of solution. J. Fluid Mech. 69, 377-403 (1975)

35. Wilkinson, W.L.: Non-Newtonian fluids: Fluid mechanics, mixing and heat transfer, pp. 61-63. Pergamon Press, London (1960)

36. Zhang, J.B., Kuang, Z.B.: Study on blood constitutive parameters in different blood constitutive equations. J. Biomech. 33, 355-360 (2000) 